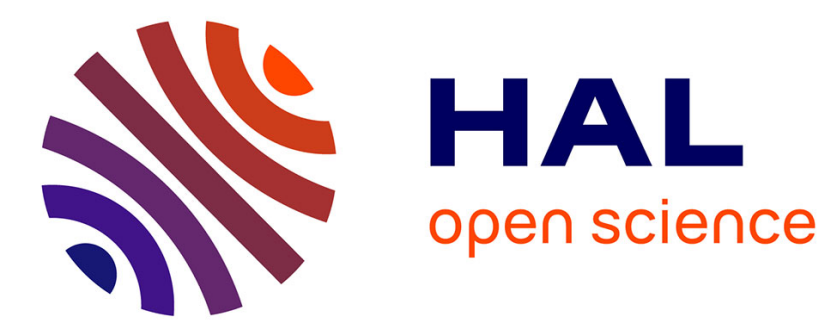

\title{
Morphological diversity and sparsity: new insights into multivariate data analysis
}

\author{
Jérôme Bobin, Jalal M. Fadili, Yassir Moudden, Jean-Luc Starck
}

\section{To cite this version:}

Jérôme Bobin, Jalal M. Fadili, Yassir Moudden, Jean-Luc Starck. Morphological diversity and sparsity: new insights into multivariate data analysis. SPIE Wavelets XII, 2007, San Diego, United States. 10.1117/12.731589 . hal-00196742

\section{HAL Id: hal-00196742 \\ https://hal.science/hal-00196742}

Submitted on 18 May 2015

HAL is a multi-disciplinary open access archive for the deposit and dissemination of scientific research documents, whether they are published or not. The documents may come from teaching and research institutions in France or abroad, or from public or private research centers.
L'archive ouverte pluridisciplinaire HAL, est destinée au dépôt et à la diffusion de documents scientifiques de niveau recherche, publiés ou non, émanant des établissements d'enseignement et de recherche français ou étrangers, des laboratoires publics ou privés. 


\title{
Morphological diversity and sparsity: new insights into multivariate data analysis
}

\author{
J. Bobin ${ }^{a}$, J. Fadili ${ }^{b}$, Y. Moudden ${ }^{a}$ and J.-L. Starck ${ }^{a}$ \\ ${ }^{a}$ DAPNIA/SEDI-SAP, Service d'Astrophysique, \\ CEA/Saclay, 91191 Gif sur Yvette, France \\ ${ }^{b}$ GREYC CNRS UMR 6072, Image Processing Group, ENSICAEN 14050, Caen \\ Cedex, France
}

Keywords: Morphological diversity, sparsity, overcomplete representation, curvelets, wavelets, multichannel data, blind source separation, denoising, inpainting

\begin{abstract}
Over the last few years, the development of multi-channel sensors motivated interest in methods for the coherent processing of multivariate data. From blind source separation (BSS) to multi/hyper-spectral data restoration, an extensive work has already been dedicated to multivariate data processing. Previous work ${ }^{1}$ has emphasized on the fundamental role played by sparsity and morphological diversity to enhance multichannel signal processing.

Morphological diversity ${ }^{2,3}$ has been first introduced in the mono-channel case to deal with contour/texture extraction. The morphological diversity concept states that the data are the linear combination of several so-called morphological components which are sparse in different incoherent representations. In that setting, piecewise smooth features (contours) and oscillating components (textures) are separated based on their morphological differences assuming that contours (respectively textures) are sparse in the Curvelet representation (respectively Local Discrete Cosine representation).

In the present paper, we define a multichannel-based framework for sparse multivariate data representation. We introduce an extension of morphological diversity to the multichannel case which boils down to assuming that each multichannel morphological component is diversely sparse spectrally and/or spatially. We propose the Generalized Morphological Component Analysis algorithm (GMCA) which aims at recovering the so-called multichannel morphological components. Hereafter, we apply the GMCA framework to two distinct multivariate inverse problems : blind source separation (BSS) and multichannel data restoration. In the two aforementioned applications, we show that GMCA provides new and essential insights into the use of morphological diversity and sparsity for multivariate data processing. Further details and numerical results in multivariate image and signal processing will be given illustrating the good performance of GMCA in those distinct applications.
\end{abstract}

\section{INTRODUCTION}

In the multichannel data setting, ${ }^{4} m$ observations $\left\{x_{j}\right\}$ are often modelled as a function of the linear combination of $n$ so-called sources $\left\{s_{i}\right\}$. In a matrix formulation (each datum is a line of the data matrix $\mathbf{X})$, the general multichannel model is as follows:

$$
\mathbf{X}=\mathcal{F}(\mathbf{A S})+\mathbf{N}
$$

where $\mathbf{A}$ is the so-called mixing matrix and $\mathbf{N}$ models instrumental noise or model imperfections; $\mathcal{F}$ is a mapping from the data space to itself. In this paper we introduce solutions to various inverse problems in the field of multichannel data analysis:

Further author information: Send correspondence to jerome.bobin@cea.fr 
1. Blind Source Separation (BSS) : in the BSS setting, the mapping $\mathcal{F}$ is the identity $($ ie $\mathcal{F}(\mathbf{X})=\mathbf{X})$ : $\overline{\mathbf{X}}=\mathbf{A S}$. Solving the BSS issue boils down to estimating the mixing matrix $\mathbf{A}$ and the sources $\mathbf{S}$.

2. Multichannel image denoising : the mapping $\mathcal{F}$ is also the identity.

3. Multichannel image inpainting : the mapping $\mathcal{F}$ is such that the observed data $\mathbf{Y}$ verifies: $\mathbf{Y}=$

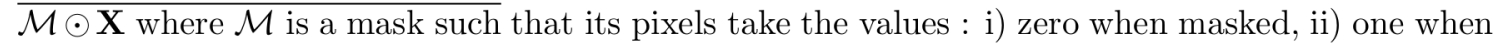
observed. The symbol $\odot$ means the mask $\mathcal{M}$ is applied pixelwise to the data $\mathbf{X}$. We also assume that no additional noise $\mathbf{N}$ perturbs the data.

Note that different choices for $\mathcal{F}$ lead to important inverse problems such as deconvolution or superresolution.

The solutions we propose rely on promising sparsity-based approaches. In the last decade sparsity has been one of the leading concept in a wide range of signal processing applications (restoration ${ }^{5}$, feature extraction $^{6}$, source separation ${ }^{1,7,8}$, to name only a few). In a wide range of applications and viewpoints, researchers have advocated the use of overcomplete signal representations. Indeed, the attractiveness of redundant signal representations relies on their ability to sparsely represent a large class of signals. Furthermore, handling very sparse signal representations allows more flexibility and entails effectiveness in many signal processing tasks (restoration, separation, compression, estimation, etc). In general, a line vector signal $x \in \mathbb{R}^{1 \times t}$ is assumed to be the linear combination of $T>t$ signal waveforms (atoms) $\left\{\phi_{i}\right\}_{i=1, \cdots, T}: x=\sum_{i=1} \alpha_{i} \phi_{i}$, where $\alpha_{i}=\left\langle x, \phi_{i}\right\rangle$ (the scalar product between $x$ and $\phi_{i}$ ) are called the decomposition coefficients of $x$ in the dictionary $\mathbf{\Phi}=\left[\phi_{1}^{T}, \cdots, \phi_{T}^{T}\right]^{T}$ (the $T \times t$ matrix whose lines are the atoms normalized to a unit $\ell_{2}$-norm).

In neuroscience, the mammalian visual system has been shown to be probably in need of overcomplete representation. ${ }^{9}$ In signal processing, both theoretical and practical arguments ${ }^{10,11}$ have supported the use of overcompleteness which entails more flexibility in representation and effectiveness at many image processing tasks. Nonetheless, handling overcomplete representations is clearly an ill-posed problem owing to elementary linear algebra.

We extended previously ${ }^{12}$ the overcomplete sparse decomposition issue to the multichannel case. In that context, we then introduce in Section 2.2 a new sparse decomposition algorithm coined Generalized Morphological Component Analysis (GMCA) devised for overcomplete dictionaries built as a union of bases. In Section 3 we show that the GMCA provides an effective analysis tool for providing astounding solutions to the aforementioned inverse problems.

\section{GENERALIZED MORPHOLOGICAL COMPONENT ANALYSIS}

\subsection{Sparse multichannel representation}

In the multichannel setting, the data $\mathbf{X}$ live in the tensor product space of $\mathbb{R}^{m}$ and $\mathbb{R}^{t}: \mathbf{X} \in \mathbb{R}^{m} \otimes \mathbb{R}^{t}$, where $m$ is the number of channels. Multichannel data are often made of $m$ "observations" or channels $\left\{\mathbf{X}_{i}\right\}_{i=1, \cdots, m}$ lying in $\mathbb{R}^{t}$. For convenience, we will use the following matrix notation :

$$
\mathbf{X}=\left[\begin{array}{c}
\mathbf{X}_{1} \\
\vdots \\
\mathbf{X}_{m}
\end{array}\right]
$$

where each channel $\left\{\mathbf{X}_{i}\right\}_{i=1, \cdots, m}$ is a $1 \times t$ line vector. A multichannel representation is no more than a set of vectors living in $\mathbb{R}^{m} \otimes \mathbb{R}^{t}$. For instance, a basis of $\mathbb{R}^{m} \otimes \mathbb{R}^{t}$ can be the tensor product of a basis of $\mathbb{R}^{m}$ (say $\boldsymbol{\Phi}_{A}$ ) with a basis of $\mathbb{R}^{t}$ (say $\boldsymbol{\Phi}_{S}$ ). Projecting $\mathbf{X}$ on such multichannel basis $\mathbf{\Phi}=\boldsymbol{\Phi}_{A} \otimes \boldsymbol{\Phi}_{S}$ is just done as follows :

$$
\alpha=\boldsymbol{\Phi}_{A}^{T} \mathbf{X} \boldsymbol{\Phi}_{S}^{T}
$$


For the sake of clarity, we will rewrite the previous projection as : $\alpha=\boldsymbol{\Phi}_{A}^{T} \mathbf{X} \boldsymbol{\Phi}_{S}^{T} \equiv \mathbf{X} \boldsymbol{\Psi}^{T}$. When a pseudo-inverse is applied, the same observation yields :

$$
\begin{aligned}
\alpha & =\left(\boldsymbol{\Phi}_{A}^{T} \boldsymbol{\Phi}_{A}\right)^{-1} \boldsymbol{\Phi}_{A}^{T} \mathbf{X} \boldsymbol{\Phi}_{S}^{T}\left(\boldsymbol{\Phi}_{S} \boldsymbol{\Phi}_{S}^{T}\right)^{-1} \\
& \equiv \mathbf{X} \boldsymbol{\Psi}^{T}\left(\boldsymbol{\Psi} \boldsymbol{\Psi}^{T}\right)^{-1}
\end{aligned}
$$

Let $\psi_{\gamma=\{i, j\}}=\phi_{A, i} \otimes \phi_{S, j}$ be an atom (i.e. element) of the multichannel dictionary $\boldsymbol{\Psi}$. This atom can be written in matrix form as : $\psi_{\gamma=\{i, j\}}=\phi_{A, i}^{T} \phi_{S, j}$ which is a $m \times t$ rank-1 matrix. The scalar product between two multichannel atoms is such that ${ }^{*}:\left\langle\psi_{\gamma=\{i, p\}}, \psi_{\gamma^{\prime}=\{j, q\}}\right\rangle=\left\langle\phi_{A, i}, \phi_{A, j}\right\rangle\left\langle\phi_{S, p}, \phi_{S, q}\right\rangle$. Handling subsets of elements that belong to $\Psi$ will also be needed. Let $\Lambda=\left\{\left\{i_{1}, j_{1}\right\}, \cdots,\left\{i_{m^{\prime}}, j_{t^{\prime}}\right\}\right\}$ be a set of index couples. Extracting the atoms of $\boldsymbol{\Psi}$ whose indices are the elements of $\Lambda$ is written $[\Psi]_{\Lambda}$. This notation will be useful for defining the support of a signal $\mathbf{X}$ in $\mathbf{\Psi}$. Assume that $\mathbf{X}$ is $K$-sparse in $\boldsymbol{\Psi}$ then $\mathbf{X}=\sum_{\gamma \in \Lambda_{x}} \alpha_{\gamma} \psi_{\gamma}$ where $[\mathbf{\Psi}]_{\Lambda_{x}}$ (or equivalently $\Lambda_{x}$ ) is the support of $\mathbf{X}$. In the next, the Froebenius or Hilbert-Schmidt norm of a matrix $\mathbf{X}$ is $\|\mathbf{X}\|^{2}=$ Trace $\left(\mathbf{X}^{T} \mathbf{X}\right)$. The $\ell_{1}$ norm of $\mathbf{X}$ is defined as the sum of the absolute value of each entry of the matrix $\mathbf{X}$. Extending the redundant representation framework to the multichannel case requires defining what a multichannel overcomplete representation $i s$. We assume that the multichannel dictionary $\boldsymbol{\Psi}$ at hand is the tensor product of a spectral dictionary $\mathbf{\Phi}_{\mathbf{A}}(m \times n$ matrix $)$ and a spatial or temporal dictionary $\boldsymbol{\Phi}_{\mathbf{S}}(T \times t$ matrix). Each atom of $\boldsymbol{\Psi}$ is then the tensor product of an atomic spectrum $\phi_{A}$ and a spatial elementary signal $\phi_{S}$ :

$$
\forall\{i, j\} \in\{1, \cdots, n\} \times\{1, \cdots, T\}, \quad \psi_{i j}=\phi_{A, i}^{T} \otimes \phi_{S, j}
$$

which is the $m \times t$ matrix $\phi_{A, i}^{T} \phi_{S, j}$.

Let's assume that the data $\mathbf{X}$ are $K$-sparse in $\mathbf{\Psi}$. Hence, $\mathbf{X}$ is the linear combination of $K$ multichannel atoms :

$$
\mathbf{X}=\sum_{\gamma \in \Lambda_{x}} \alpha_{\gamma} \psi_{\gamma}
$$

where $\operatorname{Card}\left(\Lambda_{x}\right)=K$.

\subsection{The GMCA framework}

In a slightly different context, contour/texture image decomposition techniques exploiting sparsity in overcomplete representations have been introduced in the monochannel case. ${ }^{6,13}$ In this monochannel setting, the data $x$ are assumed to be the linear combination of $D$ so-called morphological components:

$$
x=\sum_{i=1}^{D} \varphi_{i}
$$

where each morphological component $\varphi_{i}$ is sparse in a specific basis/representation $\boldsymbol{\Phi}_{\mathrm{i}}$. The whole signal $x$ is then equivalently sparse in $\mathbf{\Phi}=\left[\mathbf{\Phi}_{1}, \cdots, \mathbf{\Phi}_{\mathbf{D}}\right]$. An iterative thresholding-based algorithm coined Morphological Component Analysis (MCA) has been introduced ${ }^{13}$ to retrieve the so-called morphological components based on the incoherence between the representations $\left\{\boldsymbol{\Phi}_{\mathbf{i}}\right\}_{i=1, \cdots, D}$. Following the GMCA paradigm, ${ }^{4}$ we decompose the multichannel data $\mathbf{X}$ into $D$ morphological components which are sparse in a set of $D$ different multichannel representations $\left\{\boldsymbol{\Psi}_{k}\right\}_{k=1, \cdots, D}$. For the sake of simplicity, we assume that each multichannel sub-dictionary $\boldsymbol{\Psi}_{k}$ is orthonormal : $\forall\{i, j\},\left\langle\psi_{k}[i], \psi_{k}[j]\right\rangle=\delta_{i j}$ where $\psi_{k}[i]$ is the $i$-th atom of the $k$-th sub-dictionary. The data $\mathbf{X}$ are assumed to be the linear combination of $K$ multichannel atoms. We define $\Lambda_{k}$ as the support (i.e. the indices of active atoms) of $\mathbf{X}$ in the

\footnotetext{
${ }^{*}$ In fact, by standard properties of the tensor product, one can easily show that the Gram matrix of a tensor product is the tensor product of the Gram matrices. That is, $\mathbf{G}_{\boldsymbol{\Phi}}=\boldsymbol{\Phi} \boldsymbol{\Phi}^{T}=\mathbf{G}_{\boldsymbol{\Phi}_{\mathbf{A}}} \otimes \mathbf{G}_{\boldsymbol{\Phi}_{\mathbf{S}}}$.
} 
sub-dictionary $\boldsymbol{\Psi}_{k}$. As $\mathbf{X}$ is $K$-sparse in the whole dictionary, $\sum_{k=1}^{D} \operatorname{Card}\left(\Lambda_{k}\right)=K$. The data can be decomposed as follows :

$$
\mathbf{X}=\sum_{k=1}^{D} \sum_{i \in \Lambda_{k}} \alpha_{k}[i] \psi_{k}[i]
$$

We classically propose recovering the sparse decomposition of $\mathbf{X}$ in $\mathbf{\Psi}$ by solving the following $\ell_{1}$ sparse problem :

$$
\min _{\alpha_{k=1, \cdots, D}} \sum_{k=1, \cdots D}\left\|\alpha_{k}\right\|_{\ell_{1}} \text { s.t }\left\|\mathbf{X}-\sum_{k=1}^{D} \sum_{i \in \Lambda_{k}} \alpha_{k}[i] \psi_{k}[i]\right\|<\epsilon
$$

where $\alpha_{k}$ is the concatenation of all $\left\{\alpha_{k}[i]\right\}_{k, i}$. Problem (10) can be rewritten in its augmented Lagrangian form:

$$
\min _{\alpha_{k=1, \cdots, D}}\left\|\mathbf{X}-\sum_{k=1}^{D} \sum_{i \in \Lambda_{k}} \alpha_{k}[i] \psi_{k}[i]\right\|^{2}+2 \lambda \sum_{k=1, \cdots D}\left\|\alpha_{k}\right\|_{\ell_{1}}
$$

These two problems are equivalent under an appropriate correspondence of parameters; that is, for a given $\epsilon$, there exists a bijection $\lambda=f(\epsilon)$ such that the two problems share the same solution. As Equation (9) can be recast as a linear combination of $D$ morphological components : $\mathbf{X}=\sum_{k=1}^{D} \varphi_{k}$, the GMCA algorithm iteratively estimates each morphological component $\varphi_{k}$ by alternating between components in a Block-coordinate way. ${ }^{14}$ Each vector of coefficients $\left\{\alpha_{k}\right\}_{k}$ is then estimated as follows :

$$
\alpha_{k}=\arg \min _{\alpha_{k}}\left\|\mathbf{R}_{k}-\sum_{i \in \Lambda_{k}} \alpha_{k}[i] \psi_{k}[i]\right\|^{2}+2 \lambda\left\|\alpha_{k}\right\|_{\ell_{1}}
$$

where $\mathbf{R}_{k}=\mathbf{X}-\sum_{p \neq k} \sum_{i \in \Lambda_{p}} \alpha_{p}[i] \psi_{p}[i]$ is a residual term. Recall that for multichannel data, we write $\beta_{k}[i]=\mathbf{X} \psi_{k}[i]^{T}=\phi_{A, \gamma_{1}} \mathbf{X} \phi_{S, \gamma_{2}}^{T}$, where $\psi_{k}[i]=\phi_{A, \gamma_{1}}^{T} \otimes \phi_{S, \gamma_{2}}$. For the sake of clarity we define $\beta=\mathbf{X} \mathbf{\Psi}_{k}^{T}$ as the vector containing all the $\beta_{k}[i]$.

As we assume that each subdictionary $\boldsymbol{\Psi}_{k}$ is orthonormal, the problem in Equation (12) is equivalent to the following:

$$
\alpha_{k}=\arg \min _{\alpha_{k}}\left\|\mathbf{R}_{k} \boldsymbol{\Psi}_{k}^{T}-\alpha_{k}\right\|^{2}+2 \lambda\left\|\alpha_{k}\right\|_{\ell_{1}}
$$

which has a unique solution $\alpha_{k}=\Delta_{\lambda}\left(\mathbf{X}_{k} \mathbf{\Psi}_{k}^{T}\right)$ known as soft-thresholding with threshold $\lambda$ as follows:

$$
\Delta_{\lambda}(u[i])=\left\{\begin{array}{cc}
0 & \text { if } u[i]<\lambda \\
u[i]-\lambda \operatorname{sign}(u[i]) & \text { if } u[i] \geq \lambda
\end{array}\right.
$$

For a fixed $\lambda$, GMCA selects groups of atoms based on their scalar product with the residual $\mathbf{R}_{k}$. Allowing GMCA to select new atoms is made by decreasing the threshold $\lambda$ at each iteration. The GMCA algorithm is summarized below:

1. Set the number of iterations $I_{\max }$ and threshold $\lambda^{(0)}$

2. While $\lambda^{(h)}$ is higher than a given lower bound $\lambda_{\min }$

For $k=1, \cdots, D$

- Compute the residual term $\mathbf{R}_{k}^{(h)}$ assuming the current estimates of $\varphi_{p \neq k}, \tilde{\varphi}_{p \neq k}^{(h-1)}$ are fixed:

$\mathbf{R}_{k}^{(h)}=\mathbf{X}-\sum_{p \neq k} \tilde{\alpha}_{p}^{(h-1)} \boldsymbol{\Psi}_{p}$

- Estimate the current coefficients of $\tilde{\varphi}_{i k}^{(h)}$ by thresholding with threshold $\lambda^{(h)}$ :

$\tilde{\alpha}_{k}^{(h)}=\Delta_{\lambda(h)}\left(\mathbf{R}_{k}^{(h)} \mathbf{\Psi}_{k}^{T}\right)$

- Get the new estimate of $\varphi_{i k}$ by reconstructing from the selected coefficients $\tilde{\alpha}_{i k}^{(h)}$ :

$\tilde{\varphi}_{k}^{(h)}=\tilde{\alpha}_{k}^{(h)} \boldsymbol{\Psi}_{k}$

3. Decrease the threshold $\lambda^{(h)}$ following a given strategy 
We previously ${ }^{12}$ introduced an effective way of managing the threshold $\lambda$ coined mMOM (for multichannel "Mean Of Max").

\section{APPLICATIONS}

In the following applications, we will emphasize on prevalent inverse problems in multichannel image processing: i) blind source separation (BSS), ii) multichannel image restoration and multichannel image inpainting. In the BSS context, the usual notations involve the so-called sources $\mathbf{S}$ : if we define $\alpha_{\mathbf{S}}$ the matrix (obtained from a reordering of the coefficients $\left\{\alpha_{\gamma}\right\}$ ) such that $\mathbf{X}=\mathbf{A} \alpha_{\mathbf{S}} \boldsymbol{\Phi}_{\mathbf{S}}$; then the sources are defined as follows $\mathbf{S}=\alpha_{\mathbf{S}} \boldsymbol{\Phi}_{\mathbf{S}}$. Each individual source is obtained as : $s_{i}=\alpha_{S i} \boldsymbol{\Phi}_{\mathbf{S}}$ where $\alpha_{\mathbf{S}}$ is the $i$-th line of $\alpha_{\mathbf{S}}$. In the next Section, we illustrate the ability of GMCA to provide an effective sparsity-based framework for solving several multichannel inverse problems.

\subsection{Blind source separation}

\subsubsection{Principle}

In the Blind Source Separation ${ }^{4}$ (BSS) problem, the mapping $\mathcal{F}$ is the identity: $\mathbf{X}=\mathbf{A S}+\mathbf{N}$. We will fix the "spectral" dictionary $\mathbf{\Phi}_{\mathbf{A}}=\mathbf{I}$ to be the identity matrix. When the sources $\mathbf{S}$ are sparse in a dictionary $\boldsymbol{\Phi}_{\mathbf{S}}$, the data $\mathbf{X}$ are thus sparse in the multichannel dictionary $\mathbf{A} \otimes \boldsymbol{\Phi}_{\mathbf{S}}$. In a previous article $^{4}$ we devoted to BSS, we introduced a GMCA-based algorithm for Blind Source Separation. This "blind"-GMCA iterative algorithm accounts for the sparsity of the so-called sources $\mathbf{S}$ to estimate blindly both the mixing matrix $\mathbf{A}$ and the sources $\mathbf{S}$. The algorithm is as follows:

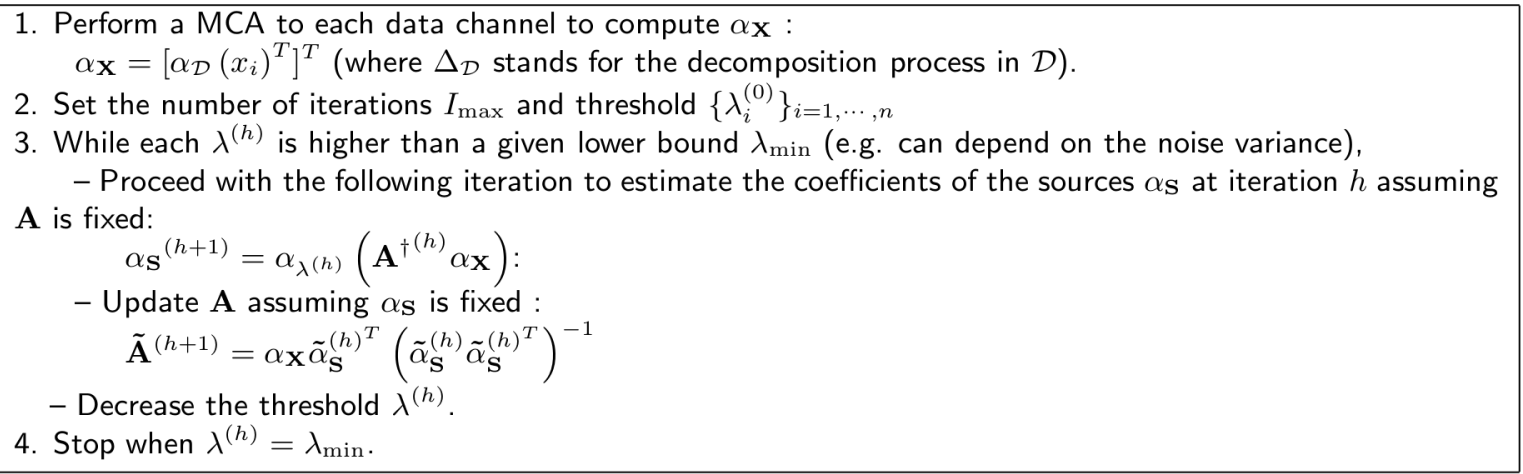

The data $\mathbf{X}$ are first decomposed in the "spatial" dictionary $\boldsymbol{\Phi}_{\mathbf{S}}$ such that $\mathbf{X}=\alpha_{\mathbf{X}} \boldsymbol{\Phi}_{\mathbf{S}}$. The mixing matrix is then estimated in the sparse domain. More details are available in a previous article ${ }^{4}$.

\subsubsection{Results}

In this section, we will compare several BSS techniques with GMCA in an image separation context. We chose 3 different reference BSS methods:

- JADE : the well-known ICA (Independent Component Analysis) based on fourth-order statistics. ${ }^{15}$

- Relative Newton Algorithm : the separation technique we already mentioned. This seminal work ${ }^{16}$ paved the way for sparsity in Blind Source Separation. In the next experiments, we used the Relative Newton Algorithm (RNA) on the data transformed by a basic orthogonal bidimensional wavelet transform (2D-DWT). 
- EFICA : this separation method improves the FastICA algorithm ${ }^{17}$ for sources following generalized Gaussian distributions. We also applied EFICA on data transformed by a 2D-DWT where the assumptions on the source distributions is appropriate.

Figure 1 shows the original sources (top pictures) and the 2 mixtures (bottom pictures). The original sources $s_{1}$ and $s_{2}$ have a unit variance. The matrix $\mathbf{A}$ that mixes the sources is such that $x_{1}=$ $0.25 s_{1}+0.5 s_{2}+n_{1}$ and $x_{2}=-0.75 s_{1}+0.5 s_{2}+n_{2}$ where $n_{1}$ and $n_{2}$ are Gaussian noise vectors (with decorrelated samples) such that the SNR equals $10 \mathrm{~dB}$. The noise covariance matrix $\boldsymbol{\Sigma}_{\mathbf{N}}$ is diagonal.

The comparisons we carry out here are twofold: (i) we evaluate the separation quality in terms of
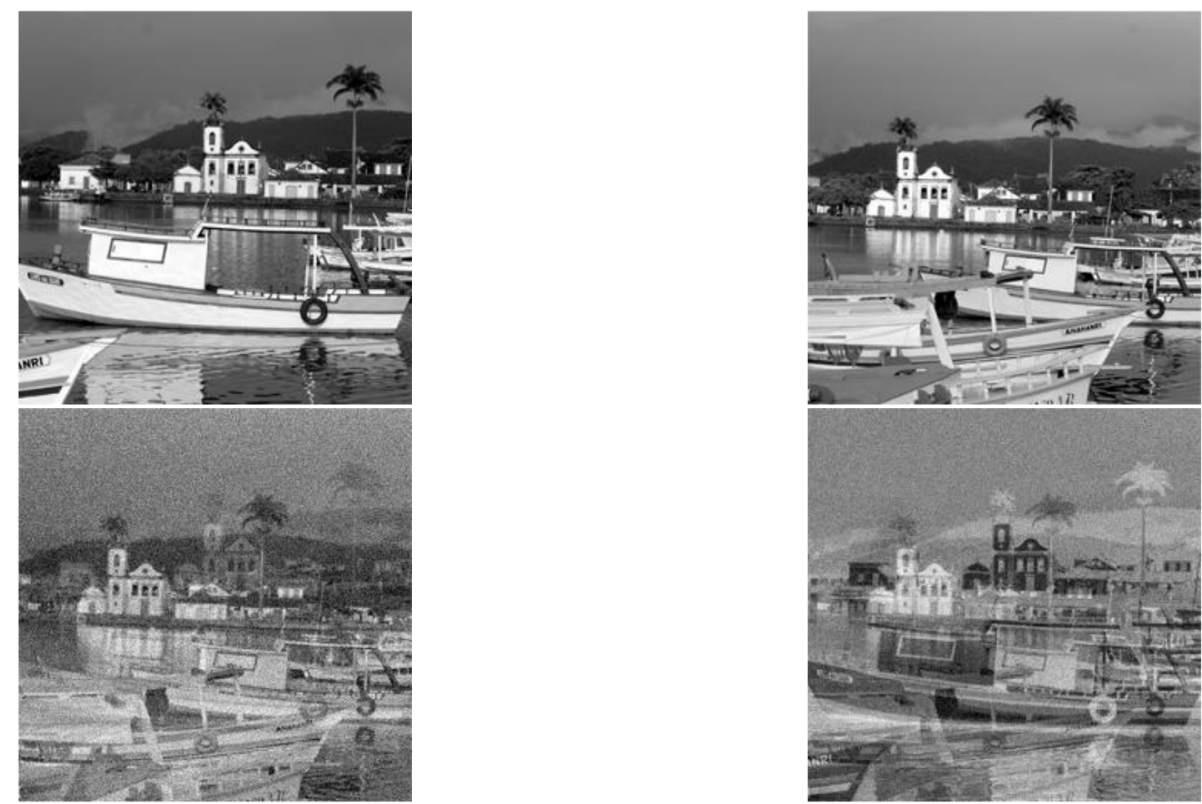

Figure 1. Top : the $256 \times 256$ source images. Bottom : two different mixtures. Gaussian noise is added such that the SNR is equal to $10 \mathrm{~dB}$.

correlation coefficient between the original and estimated sources as the noise variance varies; (ii) as the estimated sources are also perturbed by noise, correlation coefficients are not always very sensitive to separation errors, we also assess the performances of each method by computing the mixing matrix criterion $\Delta_{\mathbf{A}}{ }^{4}$. The GMCA algorithm was computed with the union of a Fast Curvelet Transform (available online $^{18}$ ) and a Local Discrete Cosine Transform (LDCT). The union of the curvelet transform and LDCT are often well suited to a wide class of "natural" images.

Figure 2 portrays the evolution of the correlation coefficient of source 1 (left picture) and source 2 (right picture) as a function of the SNR. At first glance, GMCA, RNA and EFICA are very robust to noise as they give correlation coefficients closed to the optimal value 1 . On these images, JADE behaves rather badly. It might be due to the correlation between these two sources. For higher noise levels ( SNR lower than 10dB), EFICA tends to perform slightly worse than GMCA and RNA. In our experiments, a mixing matrix-based criterion turns out to be more sensitive to separation errors and then better discriminates between the methods. Figure 3 depicts the behavior of the mixing matrix criterion as the SNR increases. Recall that the correlation coefficient was not able to discriminate between GMCA and RNA. The mixing matrix criterion clearly reveals the differences between these methods. First, it confirms the dramatic behavior of JADE on that set of mixtures. Secondly, RNA and EFICA behave rather similarly. Thirdly, GMCA seems to provide far better results with mixing matrix criterion values that are approximately 10 times lower than RNA and EFICA. 

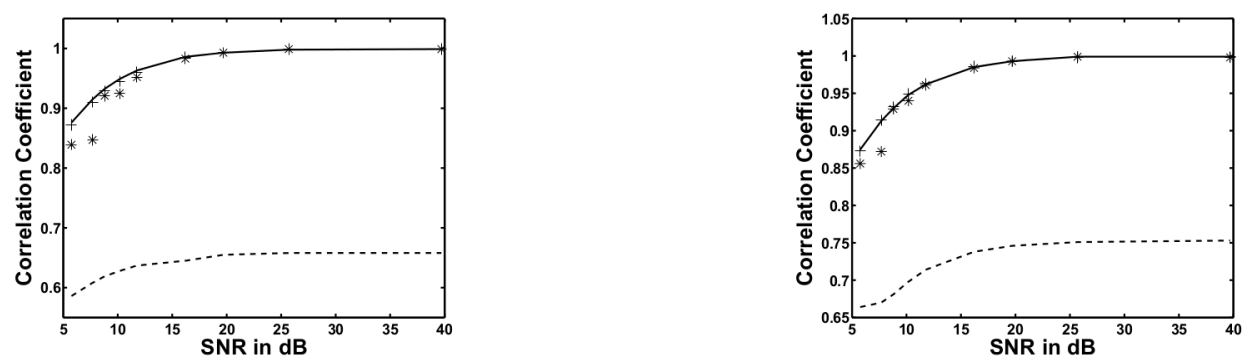

Figure 2. Evolution of the correlation coefficient between original and estimated sources as the noise variance varies: solid line: GMCA, dashed line: JADE, $(\star)$ : EFICA, $(+)$ : RNA. Abscissa : SNR in dB. Ordinate : correlation coefficients.

To summarize, the findings of this experiment confirm the key role of sparsity in blind source separation:

- Sparsity brings better results : remark that, amongst the methods we used, only JADE is not a sparsity-based separation algorithm. Whatever the method, separating in a sparse representation enhances the separation quality : RNA, EFICA and GMCA clearly outperform JADE.

- GMCA takes better advantage of overcompleteness and morphological diversity: RNA, EFICA and GMCA provide better separation results with the benefit of sparsity. Nonetheless, GMCA takes better advantage of sparse representations than RNA and EFICA.

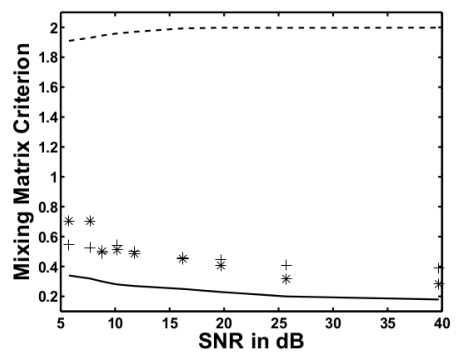

Figure 3. Evolution of the mixing matrix criterion $\Delta_{A}$ as the noise variance varies: solid line: GMCA, dashed line : JADE, $(\star)$ : EFICA, (+) : RNA. Abscissa : SNR in dB. Ordinate : mixing matrix criterion value.

\subsection{Multivariate data restoration}

In the present paper we also address some multivariate data restoration issues. More particularly, we focus on colour image restoration problems. The same restoration scheme could be easily used for any multivariate data.

\subsubsection{Denoising color images}

Up to now we emphasized on sparse blind source separation. The "spectral" dictionary is assumed to be the identity matrix: $\boldsymbol{\Phi}_{\mathbf{A}}=\mathbf{I}$. We showed ${ }^{4}$ that the stable solutions of the aforementioned blindGMCA are the sparsest in the dictionary $\boldsymbol{\Phi}_{\mathbf{S}}$. Thus it is tempting to extend GMCA to other multivalued problems such as multi-spectral data restoration.

For instance, it is intuitively appealing to denoise multivalued data (such as color images) in multichannel 
representations in which the new components are sparse in a given dictionary $\boldsymbol{\Phi}_{\mathbf{S}}$. Let's consider multivalued data stored row-wise in the data matrix $\mathbf{X}$. We assume that those multivalued data are perturbed by additive noise. Intuition tells us that it would be worth looking for a new representation $\mathbf{X}=\mathbf{A} \mathbf{S}$ such that the new components $\mathbf{S}$ are sparse in the dictionary $\mathbf{\Phi}_{\mathbf{S}}$.

We applied the blind-GMCA algorithm in the context of color image denoising ( $\mathrm{SNR}=15 \mathrm{~dB}$ ). This is illustrated in Figure 4 where the original RGB image ${ }^{\dagger}$ are shown on the left. Figure 4 in the middle shows the RGB image obtained using a classical wavelet-based denoising method on each color plane (hard-thresholding in the Undecimated Discrete Wavelet Transform (UDWT)). The blind-GMCA is computed in the curvelet domain on the RGB colour channels and the same UDWT-based denoising is applied to the sources $\mathbf{S}$. The denoised data are obtained by coming back to the RGB space via the matrix A. Figure 4 on the right shows the denoised blind-GMCA image using the same wavelet-based denoising method. Visually, denoising in the "GMCA colour space" performs better than in the RGB space. Note that GMCA was computed in the curvelet space which is known to sparsely represent piecewise smooth images with $C^{2}$ contours $^{19}$. We also applied this denoising scheme with other color
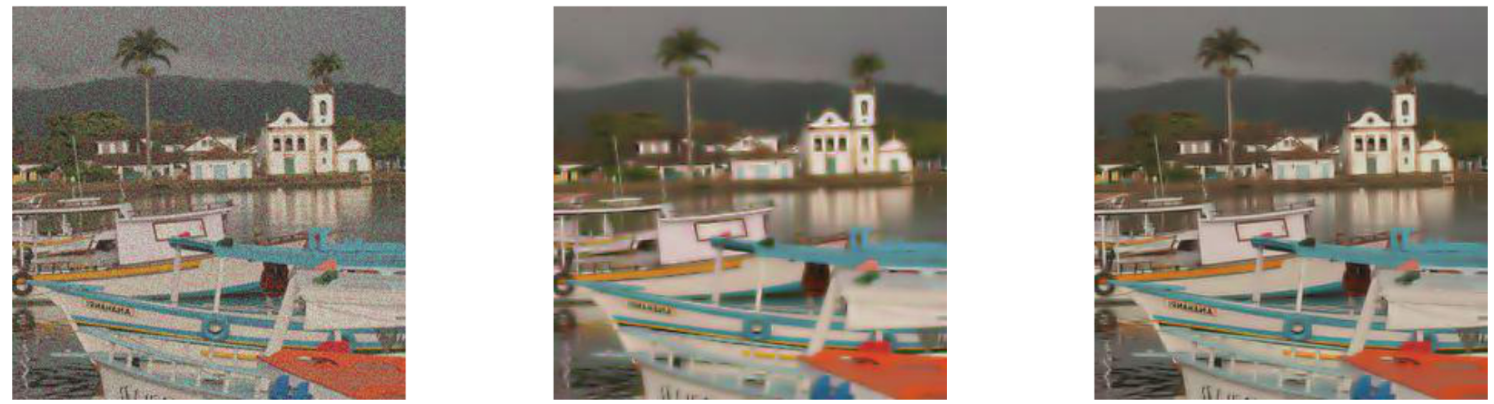

Figure 4. Left : Original $256 \times 256$ image with additive Gaussian noise. The SNR is equal to 15 dB. Middle : Wavelet-based denoising in the RGB space. Right : Wavelet-based denoising in the curvelet-GMCA space.

space representations : YUV, YCC (Luminance and chrominance spaces). We also applied JADE on the original colour images and denoised the components estimated by JADE. The question is then: would it be worth denoising in a different space (YUV, YCC, JADE or GMCA) instead of denoising in the original $R G B$ space? Figure 5 shows the SNR improvement (in $\mathrm{dB}$ ) as compared to denoising in the RGB space obtained by each method method (YUV, YCC, JADE and GMCA). Figure 5 shows that YUV and YCC representations lead to the same results. Note that the YCC colour standard is derived from the YUV one. With this particular colour image, JADE gives satisfactory results as it can improve denoising up to $1 \mathrm{~dB}$. Finally, as expected, a sparsity-based representation such as GMCA provides better results. Here, GMCA enhances denoising up to $2 \mathrm{~dB}$. This series of tests confirms the visual impression that we get from Figure 4. Note that such "GMCA colour space" is adaptive to the data.

\subsubsection{Steps ahead : learning the sparse representation :}

In this section, we consider the particular case of colour image inpainting. In this setting, the mapping $\mathcal{F}$ is no more the identity. Indeed, as data are missing, the observed data $\mathbf{Y}$ are such that : $\mathbf{Y}=\mathcal{M} \odot \mathbf{X}$ where $\mathcal{M}$ is a mask such that its pixels take the values : i) zero when masked, ii) one when observed. The symbol $\odot$ means the mask $\mathcal{M}$ is applied pixelwise to the data $\mathbf{X}$. We also assume that no additional noise $\mathbf{N}$ perturbs the data. The "spectral" dictionary is assumed to be the identity matrix: $\boldsymbol{\Phi}_{\mathbf{A}}=\mathbf{I}$. The data $\mathbf{X}$ are made of 3 observed channels corresponding to each colour layer (for instance red, green and blue). We saw previously that restoring colour image in a different colour (i.e. YUV) may sometimes enhance the restoration performance.

In a previous article ${ }^{12}$ we propose recovering masked colour images using GMCA by seeking the colour

\footnotetext{
${ }^{\dagger}$ All colour images can be downloaded at http://perso.orange.fr/jbobin/gmca2.html.
} 


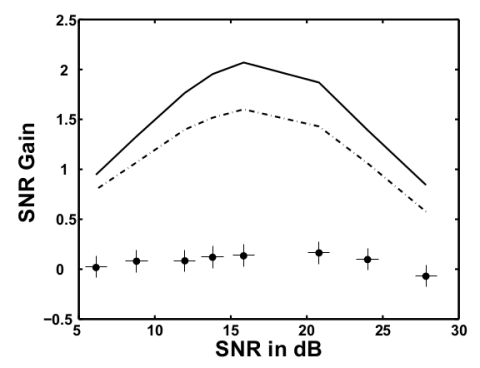

Figure 5. Denoising color images : how GMCA can improve multivariate data restoration. Abscissa : Mean SNR in dB. Ordinate : Gain in terms of SNR in dB compared to a denoising process in the RGB color space. Solid line: GMCA, dashed-dotted line: JADE, 'ø' YUV, '+': YCC.

space in which the data $\mathbf{X}$ are sparse. Assume that the data $\mathbf{X}$ are represented in the RGB space and let $\mathbf{S}$ be such that : $\mathbf{X}=\mathbf{A S}=\sum_{i=1}^{3} a^{i} s_{i}$ where $a^{i}$ is the $i$-th column of $\mathbf{A}$ and $s_{i}$ is the $i$-th line of $\mathbf{S}$. The $3 \times 3$ matrix $\mathbf{A}$ changes the colour space in which the data are represented. Adapting the colour space to the data then amounts to estimate an "optimal" matrix A.

The GMCA algorithm is then adapted such that at each iteration, each column $a^{i}$ of the colour space matrix $\mathbf{A}$ is updated by its least-squares estimate ${ }^{12}$ :

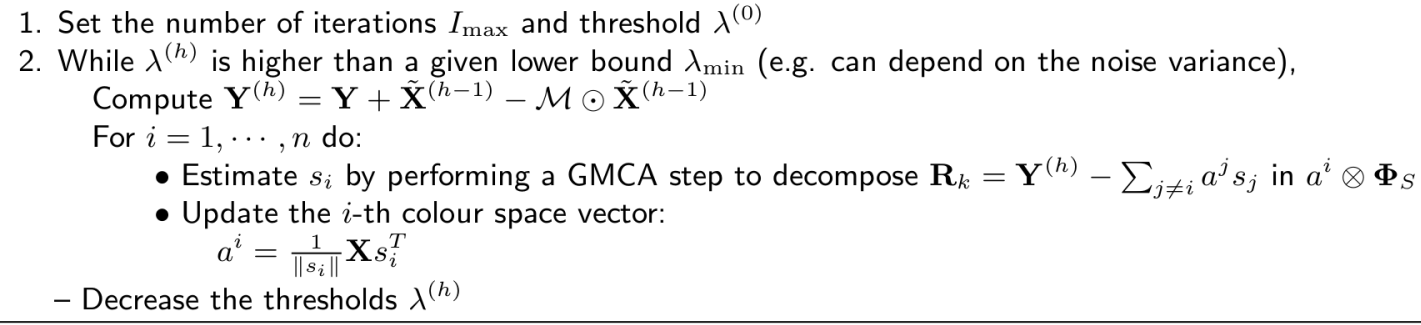

Note that when the update of the mixing matrix is omitted, the algorithm is similar to the multichannel extension of the MCA-based inpainting method introduced by Fadili ${ }^{20}$ et al. The top-left picture in Figure 6 shows the original Barbara colour image. The top-right picture depicts the masked colour image where $90 \%$ of the colour pixels are missing. The bottom-left picture portrays the recovered image using GMCA in the original RGB colour space (which amounts to perform a monochannel MCAbased inpainting on each channel). The last bottom-right picture shows the recovered image with the colour space-adaptive GMCA algorithm. The zoom on the recovered images in Figure 7 shows that adapting the colour space avoids chromatic abberrations and hence produces a better visual result. This visual impression is quantitatively confirmed by SNR measure, where the colour space-adaptive GMCA improves the SNR by $1 \mathrm{~dB}$.

\section{CONCLUSION}

The use of overcomplete representations and sparsity have emerged as an effective analysis tool in a wide range of applications. However, decomposing a signal in a redundant dictionary is theoretically challenging. We extended the sparse decomposition framework to the multichannel setting. Furthermore, designing a fast and effective decomposition algorithm is also a harsh task. We also introduced a fast and practical decomposition algorithm coined Generalized Morphological Component Analysis (GMCA). We extended GMCA to account for Blind Source Separation issues and adaptive multivariate data restoration 

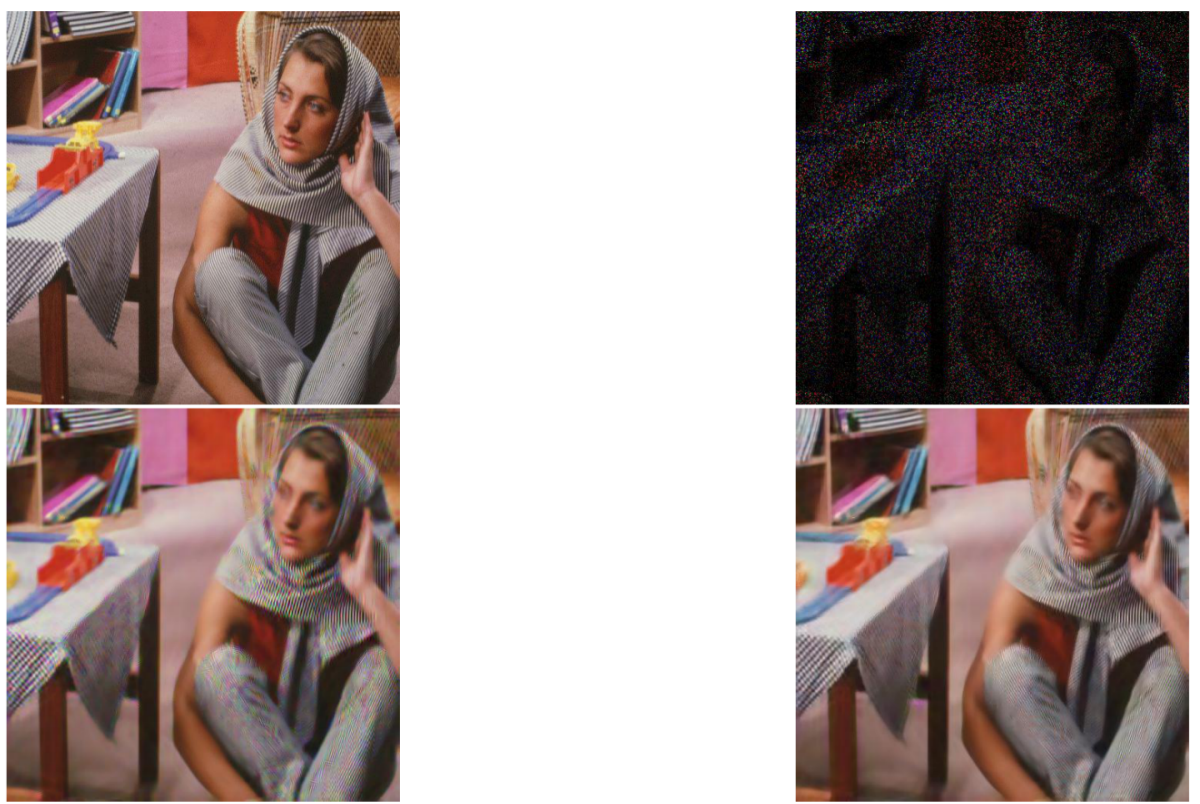

Figure 6. Recovering colour images - top-left original colour image. Top-right : masked image - $90 \%$ of the colour pixels are missing. Bottom - left : recovered image using the original GMCA algorithm. Bottom - right : recovered image using the adaptive GMCA algorithm.

for which it provides good performances. GMCA then provides an effective tool for analyzing sparse multivariate data. Further work will focus on hyperspectral image analysis.

\section{REFERENCES}

1. J. Bobin, Y. Moudden, J.-L. Starck, and M. Elad, "Morphological diversity and source separation," IEEE Signal Processing Letters 13(7), pp. 409-412, 2006.

2. M. Elad, J.-L. Starck, D. Donoho, and P. Querre, "Simultaneous cartoon and texture image inpainting using morphological component analysis (MCA)," ACHA, 2005. to appear.

3. J.Bobin, J-L.Starck, J.Fadili, Y.Moudden, and D.L.Donoho, "Morphological component analysis: new results.," submitted to IEEE Transactions on Image Processing - revised - available at http://perso.orange.fr/jbobin/articles/TIP_2006.pdf , 2006.

4. J.Bobin, J-L.Starck, J.Fadili, and Y.Moudden, "Sparsity and morphological diversity in blind source separation," IEEE Transactions on Image Processing - accepted - available at http://perso.orange.fr/jbobin/pubs2.html , 2007.

5. J.-L. Starck, E. J. Candès, and D. L. Donoho, "The curvelet transform for image denoising.," IEEE Transactions on Image Processing 11(6), pp. 670-684, 2002.

6. J.-L. Starck, M. Elad, and D. Donoho, "Image decomposition via the combination of sparse representations and a variational approach," IEEE Transactions on Image Processing, 2004. in press.

7. Y. Li, S.Amari, A.Cichocki, and C. Guan, "Underdetermined blind source separation based on sparse representation," IEEE Transactions on information theory 52, pp. 3139-3152, 2006.

8. M. Zibulevsky and B.B.Pearlmutter, "Blind source separation by sparse decomposition," Neural Computations 13/4, 2001.

9. Olshausen.BA and Field.DJ., "Sparse coding with an overcomplete basis set: A strategy employed by v1?," Vision Research. 37, pp. 3311-3325, 2006. 

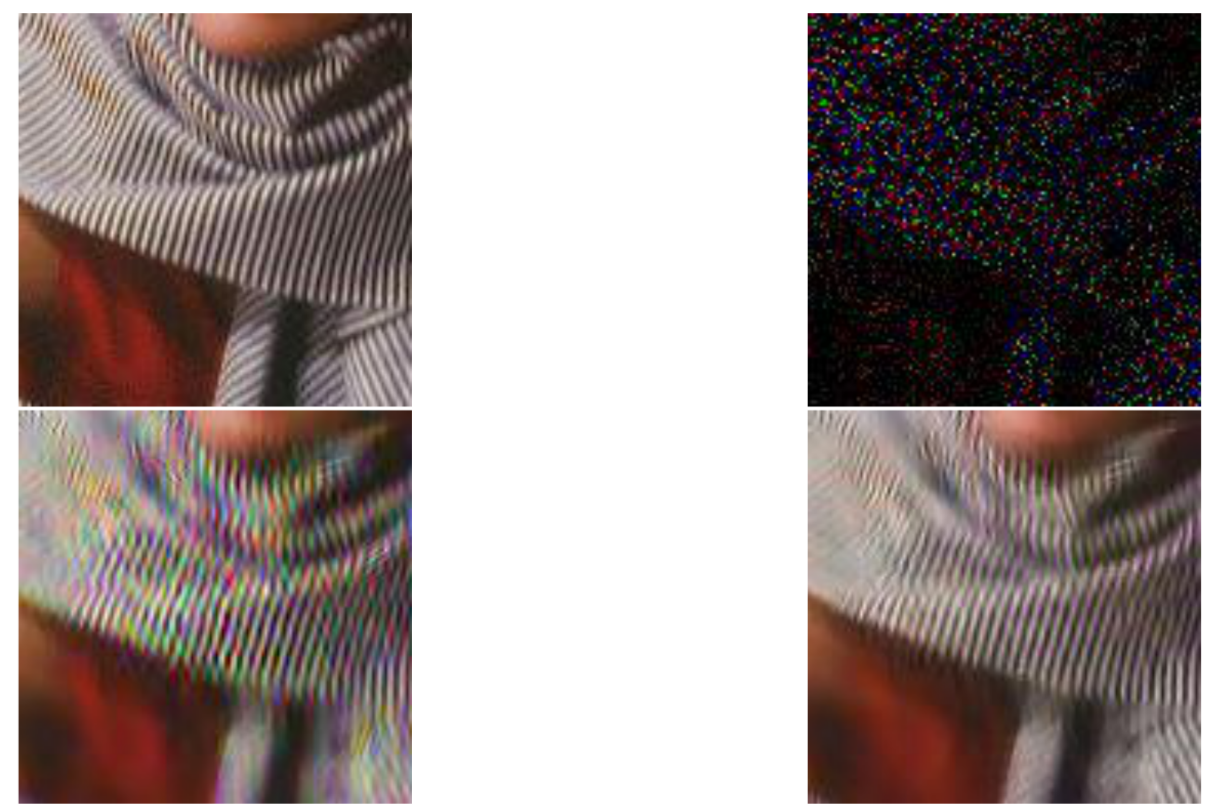

Figure 7. Zoom on recovered colour images - top-left original colour image. Top-right : masked image - 90\% of the colour pixels are missing. Bottom - left : recovered image using the original GMCA algorithm. Bottom - right : recovered image using the adaptive GMCA algorithm.

10. J.-L. Starck, E. Candès, and D. Donoho, "The curvelet transform for image denoising," IEEE Transactions on Image Processing 11(6), pp. 131-141, 2002.

11. J.-L.Starck, J.Fadili, and F.Murtagh, "The undecimated wavelet decomposition and its reconstruction," IEEE Transactions on Image Processing 16, pp. 297-309, 2007.

12. J.Bobin, Y.Moudden, J. Fadili, and J.-L. Starck, "Morphological component analysis for sparse multichannel data: Application to inpainting," Journal of Mathematical Imaging and Vision. , 2007.

13. J.-L. Starck, M. Elad, and D. Donoho, "Redundant multiscale transforms and their application for morphological component analysis," Advances in Imaging and Electron Physics 132, 2004.

14. S.Sardy, A.Bruce, and P.Tseng, "Block coordinate relaxation methods for nonparametric wavelet denoising," Journal of Computational and Graphical Statistics 9(2), pp. 361-379, 2000.

15. J.-F. Cardoso, "Blind signal separation: statistical principles," Proceedings of the IEEE. Special issue on blind identification and estimation 9, pp. 2009-2025, Oct. 1998.

16. M. Zibulevski, "Blind source separation with relative newton method," Proccedings ICA2003 , pp. 897-902, 2003.

17. P. Z.Koldovsky and E.Oja, "Efficient variant of algorithm fastica for independent component analysis attaining the cramer-rao lower bound," IEEE Transactions on neural networks 17, pp. 12651277, 2006.

18. Curvelab 2.01 for Matlab7.x. (http://www.curvelet.org/), 2006.

19. E. Candès and D. Donoho, "Curvelets," tech. rep., Statistics, Stanford University, 1999.

20. M. J. Fadili and J.-L. Starck, "Em algorithm for sparse representation - based image inpainting," IEEE International Conference on Image Processing ICIP'05 2, pp. 61-63, 2005. Genoa,Italia. 